\title{
Reliable Pain Relief But Variable Return to Play After Arthroscopic Elbow Debridement in Baseball Players
}

\author{
James F. Stenson, D.O., Quincy T. Cheesman, D.O., Jacob M. Kirsch, M.D., \\ Christopher L. Antonacci, B.S., Frank G. Alberta, M.D., and Luke S. Austin, M.D.
}

\begin{abstract}
Purpose: We sought to determine the rate of return to play (RTP) in baseball players following arthroscopic elbow debridement for the management of the symptomatic elbow. Methods: A retrospective case series with prospectively collected data via questionnaire was conducted on all baseball players who underwent an arthroscopic elbow debridement, from July, 15, 2004 to November 1, 2017. A postoperative questionnaire was released at an average 7.25 year follow-up. Data collected included age, gender, laterality, preoperative diagnosis, range of motion, duration and characterization of symptoms, visual analog scale (VAS) pain score, complications, level of play, and RTP. Results: Follow-up data were available on 18 baseball players. Average age was 19.7 years (range 16-24). Seventeen were pitchers, and 1 was a catcher. Level of play included 12 collegiate athletes, 2 high school athletes, 2 recreational athletes, 1 minor league athletes, and 1 major league athlete. Rate of RTP was $61 \%(11 / 18)$ with 6 returning to a greater level and 5 to an equal level. The length of time to RTP following surgery was most commonly within $6-8$ months $(44.4 \%, 8 / 18)$. Mean VAS pain score improved from 6.9 to $0.75(P=<.001) .27 .8 \%(5 / 18)$ had repeat surgery secondary to recurrent/persistent stiffness or heterotopic ossification. $77.8 \%(14 / 18)$ of patients rated their final outcome as either "very satisfied" $(9 / 18)$, or "satisfied" (5/18). Conclusions: Pain can reliably be relieved following arthroscopic elbow debridement in baseball players. Although patient satisfaction may be high, patients do not always return to their previous level of play. Patients must be counseled on the risk of limited postoperative athletic capacity before the time of surgery. Level of Evidence: Level IV, therapeutic case series.
\end{abstract}

\section{Introduction}

$\mathbf{E}$ lbow injuries are increasingly encountered in throwing athletes. ${ }^{1}$ Overhead throwing subjects the elbow to significant angular and torsional forces, resulting in a spectrum of overuse injuries. ${ }^{2,3}$ Repetitive overload may lead to the development of posteromedial bone spurs, ${ }^{4}$ laxity of the ulnar collateral ligament, ${ }^{5}$ intra-articular loose bodies, and capsular contracture. ${ }^{6}$

From the Department of Orthopaedics and Sports Medicine, University of Washington, Seattle, Washington, U.S.A. (J.F.S.); Rowan University School of Osteopathic Medicine, Stratford, New Jersey, U.S.A. (Q.T.C.); Boston Sports and Shoulder Center, Boston, Massachusetts, U.S.A. (J.M.K.); and Rothman Orthopaedic Institute Philadelphia, Pennsylvania, U.S.A. (C.L.A., F.G.A., L.S.A.).

Full ICMJE author disclosure forms are available for this article online, as supplementary material.

Received December 18, 2020; accepted May 20, 2021

Address correspondence to James F. Stenson, D.O., Rowan University School of Osteopathic Medicine, 42 East Laurel Rd., Stratford, NJ 08084, U.S.A.E-mail: jfstenson@gmail.com

(C) 2022 THE AUTHORS. Published by Elsevier Inc. on behalf of the Arthroscopy Association of North America. This is an open access article under the CC BY-NC-ND license (http://creativecommons.org/licenses/by-nc-nd/4.0/). 2666-061X/201967

https://doi.org/10.1016/j.asmr.2021.05.010
As a result, patients complain of pain, locking, catching, and functional limitations, inhibiting the athletic population. ${ }^{7}$

The majority of elbow injuries in athletes can be treated with rest, activity modification, and physical therapy. When nonoperative measures are ineffective, surgical intervention may be necessary to return athletes to their preinjury activity levels. ${ }^{8}$ Elbow arthroscopy is shown to be a useful tool for treating posteromedial impingement, removal of loose bodies, and osteochondritis dissecans lesions of the capitellum. ${ }^{9}$ However, there is a paucity of data assessing the rate of return to play (RTP) for baseball players who undergo an arthroscopic elbow debridement for management of the symptomatic elbow. Available data report findings based on a low level of participants.

The purpose of our study was to determine the rate of RTP in baseball players following arthroscopic elbow debridement for the management of the symptomatic elbow. We hypothesized that arthroscopic elbow debridement would allow patients to return to their previous level of play with a noticeable relief in pain and improvement in range of motion (ROM). 


\section{Methods}

This case series study was conducted in a retrospective manner. It consisted of a chart review and prospectively collected data via a questionnaire. The study was performed at a large, single-center, institution located within a large metropolitan city with more than 140 surgeons on staff. Approval from Thomas Jefferson University's Institutional Review Board was obtained prior to the initiation of this study (number no. 19D.648).

Patients were queried using CPT code 29837 (arthroscopy, elbow, surgical; debridement, limited) and 29838 (arthroscopy, elbow surgical; debridement, extensive) from July 15, 2004 to November 1, 2017. The electronic medical record was reviewed to identify patients who were labeled as baseball players. Inclusion criteria encompassed baseball players at any level who underwent arthroscopic elbow debridement in their throwing arm with a minimum of 2-year follow-up. Exclusion criteria encompassed traumatic pathology, non-sport-related elbow injuries, and patients who failed to respond to the questionnaire.

Preoperative demographic data collected included procedure date, age, gender, laterality, preoperative diagnosis, ROM, duration of symptoms, characterization of symptoms, and visual analog scale (VAS) pain score. Postoperative ROM, VAS pain score, and complications were extracted from the electronic medical record.

A RedCap questionnaire was created and distributed to patients for collection of outcome information. Patients who failed to respond to the questionnaire were contacted via telephone. Questions delineated baseball position (pitcher versus nonpitcher), history of previous trauma, history of previous surgery, characterization of symptoms, level of play (high school, recreational, college, professional) prior to injury, level of play following surgery, length of time to RTP following surgery, ability to regain ROM/accuracy/velocity following surgery, satisfaction, VAS pain score, persistence/recurrence of symptoms, need for further treatment or surgery, and complications (Appendix 1).

The primary outcome of our study was to determine the rate of RTP in baseball players following arthroscopic elbow debridement. We defined RTP as a patient's ability to compete at the same or higher level of play within 12 months following surgery. Secondary outcomes included average length of time to RTP, ability to regain ROM/accuracy/velocity, preoperative versus postoperative ROM, preoperative versus postoperative VAS pain score, rate of recurrent/persistent symptoms, rate of need for further treatment or surgery, and rate of satisfaction.

\section{Statistical Methods}

All statistical analysis was performed using R Studio (version 3.5.1, Vienna, Austria). Despite a relatively low number of study participants, the data fit a normal distribution; and thus, was analyzed with parametric measures. All continuous data were presented as means and ranges, while all categorical data were presented as cell count and percentages. Independent samples $t$-tests were used to compare preoperative and postoperative data. Statistical significance was set at $P<0.05$.

\section{Results}

Thirty-two baseball players underwent arthroscopic elbow debridement during our study period. Eighteen patients met our inclusion criteria, completed the questionnaire by phone or email, and were included for analysis in the study. The remaining 14 patients failed to complete the questionnaire and were unable to be contacted despite multiple email and telephone attempts.

The average age at time of surgery was 19.7 years (range: $16-24$ years). $100 \%(18 / 18)$ of patients were male $88.9 \%(16 / 18)$ had surgery on their right elbow while $11.1 \%(2 / 18)$ had surgery on their left elbow. $94.4 \%(17 / 18)$ of patients were pitchers, while the remaining $5.6 \%(1 / 18)$ was a catcher. The mean follow-up time from surgery to questionnaire completion was 7.25 years (range: $2.75-14.4$ years).

Level of play at time of injury was most commonly the college athlete $(66.7 \%, 12 / 18)$, followed by high school athlete $(11.1 \%, 2 / 18)$, recreational athlete $(11.1 \%, 2 / 18)$, minor league athlete $(5.56 \%, 1 / 18)$, and professional athlete $(5.56 \%, 1 / 18)$. Preoperative diagnoses were not limited to one etiology and included posteromedial impingement $(88.9 \%, 16 / 18)$, intraarticular loose body $(55.6 \%, 10 / 18)$, concomitant UCL injury $(27.8 \%, 5 / 18)$, ulnar neuritis not requiring transposition or decompression $(16.7 \%, 3 / 18)$, osteochondral dissecans lesion $(16.7 \%, 3 / 18)$, and bone spur other than posteromedial impingement $(5.6 \%, 1 / 18)$. Pain was the most common preoperative symptom $(100 \%, 18 / 18)$, followed by decreased ROM $(44.4 \%, 8 /$ $18)$, clicking and/or popping $(38.9 \%, 7 / 18)$, decreased throwing velocity $(38.9 \%, 7 / 18)$, decreased accuracy $(33.3 \%, 6 / 18)$, decreased performance $(33.3 \%, 6 / 18)$, locking $(27.8 \%, 5 / 18)$, and numbness/tingling (16.7\%, $3 / 18)$.

The primary outcome of our study was rate of RTP, defined as a patient's ability to compete at the same or higher level of play within 12 months following surgery. Our study demonstrates a $61 \%(11 / 18)$ rate of RTP with $33.3 \%(6 / 18)$ returning to a greater level of play and $27.8 \%(5 / 18)$ returning to an equal level of play. On the contrary, $33.3 \%(6 / 18)$ returned to a 
lower level of play and 5.6\% (1/18) did not RTP secondary to decreased ROM and loss of interest.

The length of time to RTP following surgery was most commonly within 7-8 months $(33.3 \%, 6 / 18)$, followed by $12+$ months $(22.2 \%, 4 / 18), 6-7$ months $(11.1 \%, 2 /$ $18), 4-5$ months $(11.1 \%, 2 / 18), 10-11$ months $(5.56 \%$, $1 / 18), 8-9$ months $(5.56 \%, 1 / 18), 5-6$ months $(5.56 \%$, $1 / 18)$, and 3-4 months $(5.56 \%, 1 / 18)$.

Patient's ability to regain elbow ROM, throwing velocity, and throwing accuracy were subjectively evaluated with the post hoc questionnaire. 55.6\% (10/18) were able to regain full ROM, $61.1 \%(11 / 18)$ were able to regain throwing velocity, and $77.8 \%$ (14/18) were able to regain throwing accuracy.

The mean functional arcs of motion preoperatively and postoperatively were assessed. The mean preoperative flexion was $138^{\circ}$ (range: $120^{\circ}-150^{\circ}$ ) and the mean postoperative flexion was $136^{\circ}$ (range: $120^{\circ}-150^{\circ}$ ). The average change in flexion was not statistically significant $(P=.456)$. The mean preoperative extension was $9^{\circ}$ (range: $0^{\circ}-45^{\circ}$ ), and the mean postoperative extension was $4^{\circ}$ (range: $-5^{\circ}$ to $\left.15^{\circ}\right)$. The average improvement in extension was not statistically significant $(P=0.066)$; however, it did demonstrate a clinical improvement. Preoperative and postoperative pronosupination was full and unchanged at $90^{\circ}$ each.

A subgroup analysis was performed to assess the change in ROM for the cohort of patients who underwent a repeat surgery $(n=5)$. The mean preoperative flexion was $137^{\circ}$ (range: $120^{\circ}-150^{\circ}$ ) and the mean postoperative flexion was $136^{\circ}$ (range: $120^{\circ}-150^{\circ}$ ). The average change in flexion was not statistically significant $(P=.157)$. The mean preoperative extension was $14^{\circ}$ (range: $0^{\circ}-45^{\circ}$ ), and the mean postoperative extension was $3^{\circ}$ (range: $\left.0^{\circ}-10^{\circ}\right)$. The average improvement in extension was not statistically significant $(P=0.872)$; however, it did demonstrate a clinical improvement.

An investigation of the VAS pain score revealed a preoperative mean of 6.9 (range: $2.6-9.5$ ) and a postoperative mean of 0.75 (range: 0-5.5). This improvement in VAS pain score was statistically significant $(P<.001)$.

$33 \%(6 / 18)$ of patients had a reoccurrence or persistence of symptoms following surgery. 33\% (6/18) of patients sustained a complication following surgery. The most common complication was stiffness, requiring three patients to have revision surgery consisting of debridement with capsular release. Two patients had an extended postoperative physical therapy course. The next common complication was heterotopic ossification, requiring two patients to have revision surgery consisting of repeat debridement and removal of loose body.

Overall, $77.8 \%(14 / 18)$ of patients rated their final outcome as either "very satisfied" (9/18) or "satisfied"
$(5 / 18)$. $11.1 \%(2 / 18)$ rated their satisfaction as "neutral" and $11.1 \%(2 / 18)$ rated their satisfaction as "dissatisfied".

\section{Discussion}

Our case series found a $61 \%$ rate of RTP with an average length of 6-8 months following surgery. Additionally, patients had improvements in extension ROM and pain scores, with an overall satisfaction rate of $77.8 \%$. Despite this, $27.8 \%$ had repeat surgery for either recurrent/persistent stiffness or heterotopic ossification.

There is a paucity of studies evaluating the outcomes of arthroscopic elbow debridement for the management of the symptomatic elbow in baseball players. Most studies either evaluate a combination of throwing and nonthrowing athletes or have a lower number of patients included. ${ }^{10-15}$ Our findings expand on the limited data on baseball players following arthroscopic elbow debridement.

The most significant finding of our study was that only $61 \%$ of patients returned to a previous or higher level of play following arthroscopic debridement. This rate of RTP is lower than what is commonly reported in the literature. ${ }^{10,12}$ Generally, arthroscopic treatment of elbow pathology has resulted in favorable RTP in overhead athletes. ${ }^{9}$ Blonna et al. noted that 23 of 26 elbows that underwent arthroscopic contracture release returned to their previous level of sport. However, only one patient was a baseball player. ${ }^{13}$ Yan et al. reported a $100 \%$ return to previous level of play following arthroscopic debridement in 36 professional athletes with radiographic evidence of osteoarthritis, two of which were baseball players. ${ }^{11}$ Matsuura et al. demonstrated a $100 \%$ rate of RTP among their cohort of 18 baseball players, six of which were pitchers and were mostly high school athletes. ${ }^{12}$ Our low RTP could be attributed to 17 of 18 of our patients being pitchers, a group that sustains elbow injuries at higher rates and require more days off to RTP compared with position players. ${ }^{16}$ Furthermore, none of the aforementioned studies have a follow-up longer than 3.5 years. Our average follow-up time was 7.25 years. It is possible with longer follow-up, we were able to capture a more accurate representation of the natural history of the disease process.

Although we report a low rate of RTP, our cohort demonstrated a significant improvement in pain. Arthroscopic elbow debridement is known to provide pain relief. For example, Yan et al. examined 36 elbows of professional athletes undergoing arthroscopic debridement of posterior osteophytes from the elbow. Sixteen patients reported excellent pain relief, 14 reported good pain relief, and 6 reported poor pain relief. $^{13}$ Furthermore, Rahussen et al. reported a statistically significant decreased average VAS pain 
score following arthroscopic elbow debridement in 16 athletes with posteromedial impingement. ${ }^{17}$ In addition to improvements in symptomatic baseball players, arthroscopic debridement of the elbow also provides pain relief in cases of degenerative arthritis and after removal of loose bodies. ${ }^{18-20}$ Multiple studies cite overall patient satisfaction after elbow debridement for post-traumatic contractures, degenerative processes, and athletic injuries. ${ }^{6,11,21,22}$ Overall, our results have mirrored the findings in the literature, both in the athletic and general population.

Complications following elbow arthroscopy are rare. Many authors report no complications following surgery in small patient cohorts. ${ }^{17,22}$ Authors that do report complications reveal transient nerve injuries to be the most common complication. ${ }^{9,23,24}$ We experienced only one subjective neuropraxia, which completely resolved over the span of 2 months. Other complications included three patients with residual stiffness and two patients with heterotopic ossification, all of which required a repeat operation. Of note, of the five patients who required reoperation, four of them resumed either equal or higher-level playing after their initial arthroscopic elbow debridement. It is not surprising that a patient will go on to develop symptoms again if they resume the activity that initially caused their disease process.

We report a $27.8 \%$ reoperation rate following initial arthroscopic debridement. Our rate of reoperation is higher than Kim et al., who noted an $8.3 \%$ rate of reoperation follow the arthroscopic removal of lateral synovial plicae in 12 elbows of throwing athletes and golfers. ${ }^{22}$ Conversely, Andrews et al. studied 72 professional baseball players who underwent either open or arthroscopic elbow surgery and reported a nearequivalent rate of reoperation at 33\%. ${ }^{10}$ Our findings may be due to baseball players placing increased stress on their elbows following surgical management in comparison to other athletes.

Reasons for reoperation included recurrent/persistent stiffness and heterotopic ossification. We hypothesize the etiology of the stiffness to be multifactorial. Access to appropriate physical therapy following elbow arthroscopy may not be equivalent in our population, which ranged from high school athletes to major league baseball athletes. Nevertheless, patients must be made aware of this risk prior to index surgical intervention.

Strengths of our paper include a population of isolated baseball players, with a high percentage $(94 \%$, 17/18) being pitchers. Our cohort represents a large number of athletes who regularly place high-impact valgus stress across their elbows. Furthermore, we have adequate follow-up with a mean duration of 7.25 years, which is substantially longer than most studies. Our data accurately capture the treatment time necessary to fully evaluate the efficacy of arthroscopic elbow debridement and the natural history of the disease process.

\section{Limitations}

Our study is not without limitations. First, we included a heterogeneity of concomitant elbow pathology treated with arthroscopic elbow debridement. It is possible our patients represent different clinical entities causing similar symptoms. The value of our findings lies in the purposeful evaluation of only baseball players. Second, our study lacked a control group and is prone to surgeon selection bias when treating baseball players with varying degrees of elbow pathology. Third, our post hoc questionnaire was completed an average of 7 years following surgery, thus, leaving our results prone to recall bias. Lastly, our sample size is relatively small, which is not unexpected, given the infrequency of elbow arthroscopy in baseball players.

\section{Conclusion}

Pain can reliably be relieved following arthroscopic elbow debridement in baseball players. Although patient satisfaction may be high, patients do not always return to their previous level of play. Patients must be counseled on the risk of limited postoperative athletic capacity before the time of surgery.

\section{References}

1. Kramer DE. Elbow pain and injury in young athletes. J Ped Orthopaed 2010;30:S7-S12.

2. Aguinaldo AL, Chambers H. Correlation of throwing mechanics with elbow valgus load in adult baseball pitchers. Am J Sports Med 2009;37(10):2043-2048.

3. Ciccotti MG, Pollack KM, Ciccotti MC, et al. Elbow injuries in professional baseball: epidemiological findings from the major league baseball injury surveillance system. Am J Sports Med 2017;45:2319-2328.

4. Limpisvasti O, El Attrache NS, Jobe FW. Understanding shoulder and elbow injuries in baseball. J Am Acad Orthop Surg 2007;15:139-147.

5. Kooima CL, Anderson K, Craig JV, Teeter DM, van Holsbeeck M. Evidence of subclinical medial collateral ligament injury and posteromedial impingement in professional baseball players. Am J Sports Med 2004;32: 1602-1606.

6. Jhan SW, Chou WY, Wu KT, Wang CJ, Yang YJ, Ko JY. Outcomes and factors of elbow arthroscopy upon returning to sports for throwing athletes with osteoarthritis. J Orthop Surg Res 2018;13:280.

7. Isa AD, Athwal GS, King GJW, MacDermid JC, Faber KJ, Arthroscopic debridement for primary elbow osteoarthritis with and without capsulectomy: A comparative cohort study. Shoulder Elbow 2018;10:223-231.

8. Griffith TB, Kercher J, Clifton Willimon S, Perkins C, Duralde XA. Elbow injuries in the adolescent thrower. Curr Rev Musculoskelet Med 2018;11:35-47. 
9. O'Holleran JD, Altchek DW. Elbow arthroscopy: Treatment of the thrower's elbow. Instr Course Lect 2006;55: 95- 107.

10. Andrews JR, Timmerman LA. Outcome of elbow surgery in professional baseball players. Am J Sports Med 1996;23: 407-413. doi:10.1177/036354659502300406.

11. Yan H, Cui GQ, Wang JQ, Yin Y, Ao YF. Arthroscopic debridement of osteoarthritic elbow in professional athletes. Chin Med J (Engl) 2011 ; 124:4223-4228.

12. Matsuura T, Iwame T, Suzue N, et al. Clinical outcome of arthroscopic treatment for posteromedial elbow impingement in adolescent baseball players. Arthroscopy 2018:34:105-110.

13. Blonna D, Lee G-C, O'Driscoll SW. Arthroscopic restoration of terminal elbow extension in high-level athletes. Am J Sports Med 2010;38:2509-2515. doi:10.1177/03635 46510376727.

14. Koh JL, Zwahlen BA, Altchek DW, Zimmerman TA. Arthroscopic treatment successfully treats posterior elbow impingement in an athletic population. Knee Surg Sports Traumatol Arthrosc 2017;26:306-31 1. doi:10.1007/s00167017-4563-1.

15. Oka Y. Debridement for osteoarthritis of the elbow in athletes. Int Orthopaed 1999;23:91-94. doi:10.1007/s002 640050315.

16. Ciccotti MC, Stull JD, Buckley PS, Cohen SB. Correlation of MRI to arthroscopy in the elbow: Thrower's elbow and ulnar collateral ligament injury. Sports Med Arthrosc Rev 2017;25:191-198.
17. Rahusen FTG, Brinkman JM, Eygendaal D. Arthroscopic treatment of posterior impingement of the elbow in athletes: A medium-term follow-up in sixteen cases. J Shoulder Elbow Surg 2009;18:279-282.

18. Ogilvie-Harris DJ, Gordon R, MacKay M. Arthroscopic treatment for posterior impingement in degenerative arthritis of the elbow. Arthroscopy 1995; 1 1:437-443.

19. Ogilvie-Harris DJ, Schemitsch E. Arthroscopy of the elbow for removal of loose bodies. Arthroscopy 1993;9:5-8.

20. Redden JF, Stanley D. Arthroscopic fenestration of the olecranon fossa in the treatment of osteoarthritis of the elbow. Arthroscopy 1993;9:14-16.

21. Cohen SB, Valko C, Zoga A, Dodson CC, Ciccotti MG. Posteromedial elbow impingement: Magnetic resonance imaging findings in overhead throwing athletes and results of arthroscopic treatment. Arthroscopy 2011;27: 1364-1370.

22. Kim DH, Gambardella RA, Elattrache NS, Yocum LA, Jobe FW. Arthroscopic treatment of posterolateral elbow impingement from lateral synovial plicae in throwing athletes and golfers. Am J Sports Med 2006;34: 438-444.

23. O'Holleran JD, Altchek DW. The thrower's elbow: Arthroscopic treatment of valgus extension overload syndrome. HSS J 2006;2:83-93.

24. Poehling GG, Whipple TL, Sisco L, Goldman B. Elbow arthroscopy: A new technique. Arthroscopy 1989;5: 222-224. 